\title{
Prevención del síndrome visual informático en docentes que realizan teletrabajo en una unidad educativa
}

Prevention of Visual Computer Syndrome in teachers who telework in an Educational Unit

Francisca Nieto Paredes. ${ }^{1}$ \& Manolo Alexander Córdova Suárez. ${ }^{2}$

Recibido: 18-07-2021 / Revisado: 30-07-2021 /Aceptado: 22-08-2021/ Publicado: 05-09-2021

DOI: https://doi.org/10.33262/anatomiadigital.v4i3.1.1918

\begin{abstract}
Resumen

Introduction. During the pandemic, Introducción. Durante la pandemia, el confinement forced teachers to telework, modifying occupational risks and therefore the consequences of their exposure. Ergonomic risk-related pathologies are the most common, even causing visual problems. Objective. To establish an ergonomic prevention plan, using the ROSA (Rapid Office Strain Assessment) method, to reduce the prevalence of Computer Visual Syndrome (SVI) in teachers who perform telework in Educational Unit A. confinamiento obligó a los docentes a realizar teletrabajo, modificando los riesgos ocupacionales y por ende las consecuencias de su exposición. Las patologías relacionadas con los riesgos ergonómicos son las más comunes, ocasionando incluso problemas visuales. Los Objetivo. Establecer un plan de prevención ergonómico, utilizando el método ROSA (Rapid Office Strain Assessment), para disminuir la Methodology. A prospective, Informático (SVI) en los docentes que correlational, quantitative qualitative realizan teletrabajo en la Unidad

\footnotetext{
1 Universidad Regional Autónoma de los Andes, Posgradista. Ambato-Ecuador. Ambato-Ecuador pg.mariafnp96@uniandes.edu.ec ORCID: (D) https://orcid.org/0000-0001-7864-9772

2 Universidad Nacional de Chimborazo, Facultad de Ingeniería, Riobamba-Ecuador, manolo.cordova@unach.edu.ec ORCID: (iD) https://orcid.org/0000-0001-6786-7926
} 
research was carried out. A 75 teachers were evaluated, exposed for six months to teleworking. For the diagnosis of the pathology, the Computer Vision Syndrome Questionnaire (CVS-Q) was used, while to determine the level of ergonomic risk to which they were exposed, an analysis was carried out with the Rapid Office Strain Assessment (ROSA) method. Results: After the application of the CVS-Q, $76 \%$ of teachers presented with SVI, with the age range with the highest prevalence being 20-35 years. On the other hand, with the initial evaluation of the ROSA method, $61.3 \%$ of teachers who present a high and very high level of risk were identified, evidencing in all cases, a daily exposure greater than 4 hours. Of these workers, 91.3\% have Computer Visual Syndrome. After the application of the ergonomic prevention plan, it was possible to reduce the level of ergonomic risk, modifying: exposure time to screen and peripherals, seat height, inclination of the backrest and lumbar support, position of the screen, brightness and reflections on the screen, use of the phone and use of the mouse. Conclusion. The adequacy of the workplace with the implementation of active breaks, ocular gymnastics, screen exposure time, correct use of peripherals, use of ergonomic chair, screen position, use of anti-reflective screen, use of ergonomic mouse, lower risk $100 \%$ ergonomic according to the ROSA method..

Keywords: Occupational health; computer visual syndrome; teleworking; prevention.
Educativa A. Metodología. Se realizó una investigación prospectiva, correlacional, cuali cuantitativa. Se evaluaron 75 docentes, expuestos durante seis meses al teletrabajo. Para el diagnóstico de la patología se utilizó el Computer Vision Syndrome Questionnaire (CVS-Q), mientras que para determinar el nivel de riesgo ergonómico al que se encontraban expuestos se realizó un análisis con el método Rapid Office Strain Assessment (ROSA). Resultados. Tras la aplicación del CVS-Q se observa un $76 \%$ de docentes que presenta SVI, siendo el rango de edad con mayor prevalencia el de 20-35 años. Por otro lado, con la evaluación inicial del método ROSA se identificó un $61.3 \%$ de docentes que presentan un nivel de riesgo alto y muy alto, evidenciándose en todos los casos, una exposición diaria mayor a 4 horas. De estos trabajadores, el $91,3 \%$ presenta Síndrome Visual Informático. Tras la aplicación del plan de prevención ergonómico se logró disminuir el nivel de riesgo ergonómico, modificando: tiempo de exposición a pantalla y periféricos, altura del asiento, inclinación del respaldo y apoyo lumbar, posición de la pantalla, brillos y reflejos en la pantalla, uso del teléfono y uso del mouse. Conclusión. La adecuación del puesto de trabajo con la implementación de pausas activas, gimnasia ocular, tiempo de exposición a pantalla, uso correcto de periféricos, Uso de silla ergonómica, posición de la pantalla, uso de pantalla antirreflejos, uso del mouse ergonómico, disminuye el riesgo ergonómico en un $100 \%$ según el método ROSA. 
Palabras clave: Salud laboral; síndrome visual informático; teletrabajo; prevención.

\section{Introducción}

Los cambios sociales y económicos consecuentes al confinamiento obligaron a las empresas a adoptar nuevas modalidades de trabajo, de manera abrupta, tales como el teletrabajo (Serra, 2020). El teletrabajo se caracteriza por el uso de las TICs (tecnologías de la información y la comunicación) fuera del espacio laboral, sin seguimiento ni verificación de los puestos de trabajo que garanticen una adecuada adaptación (Serra, 2020). A partir del 19 de marzo del 2020, fecha en que inició en confinamiento en el Ecuador y se suspendieron las actividades educativas presenciales (Ministerio de Educación del Ecuador, s.f.), los docentes de todo el país adoptaron la modalidad del teletrabajo. Por tal motivo, se vieron en la necesidad de instalar en su casa una oficina, utilizando el mobiliario y equipo informático que tenían a su alcance, sin las características ergonómicas adecuadas que prevengan los efectos perjudiciales de su uso a largo plazo (Organización Internacional del Trabajo [OIT], 2020). Estos se relacionan principalmente con la visión, el cuello, los miembros superiores, y la espalda (DiegoMas, 2015).

El Síndrome Visual Informático, o Síndrome Visual del Computador, engloba un grupo de síntomas resultantes del uso prolongado de computadoras, tabletas, lectores electrónicos y teléfonos celulares (America Optometric Association, 2021). También es conocido como Síndrome de Fatiga Ocular (SFO) o astenopia, y puede cursar con molestias oculares tales como irritación, prurito, lagrimeo, visión borrosa, así como extraoculares, siendo las más frecuentes cefalea, vértigo y dolor del cuello (Instituto de Seguridad y Salud en el Trabajo, 2020). Este síndrome está reconocido por la Organización Internacional del Trabajo (OIT) dentro del grupo de enfermedades laborales (Prado et al., 2017).

Este trabajo aplica la modificación de elementos factibles en los sitios de oficinas improvisadas por los docentes (silla, pantalla y periféricos), que se relacionan con la aparición del Síndrome Visual Informático.

\section{Metodología}

Para la obtención de los datos, se aplicó una encuesta digital a 75 docentes que han realizado teletrabajo por un lapso igual o mayor a 6 meses. La investigación cualitativa se realizó mediante el cuestionario CVS - Q, y la cuantitativa mediante la aplicación del método ROSA. Se recopilaron también datos de la edad y género de los trabajadores. Los docentes que formaron parte de la investigación lo hicieron voluntariamente, tras la sociabilización de la misma por parte del área de Salud Ocupacional de la Unidad Educativa A. 


\section{Aplicación del cuestionario Computer Vision Syndrome Questionnaire (CVS-Q)}

Existen algunos métodos que permiten determinar la presencia del Síndrome Visual Informático. De éstos, se escogió al CVS - Q, por ser una herramienta diagnóstica validada y replicable (Ver la Tabla 1), de aplicación sencilla, con preguntas fáciles de entender para los trabajadores. Además, sus resultados pueden ser comparados tras realizarse modificaciones en las condiciones de trabajo (Seguí, 2015).

Tabla 1

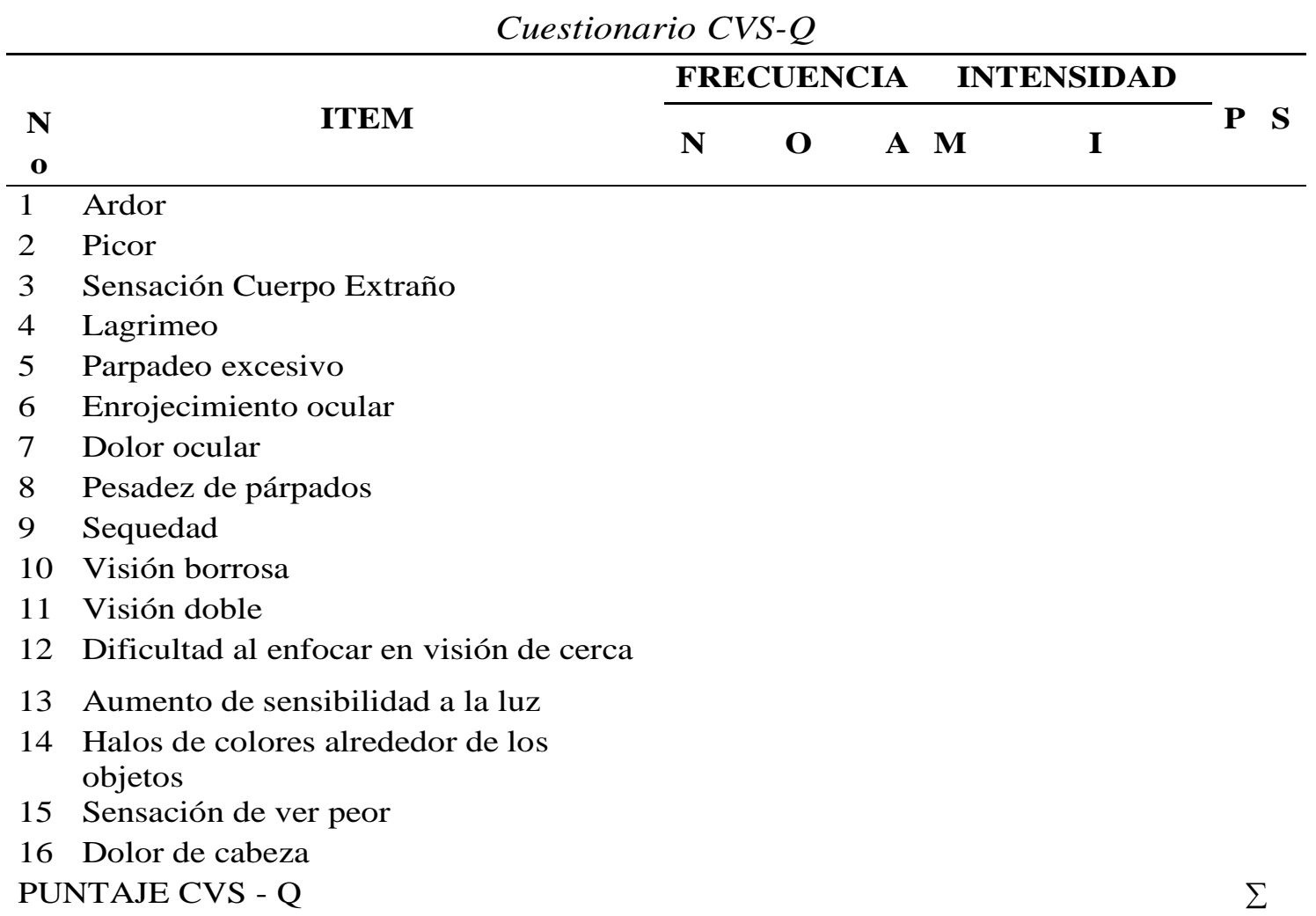

Nota: Ítems del Cuestionario Computer Vision Syndrome Questionnaire (CVS-Q). Adaptado de (Seguí, 2015). $\mathrm{N}=$ nunca, $\mathrm{O}=$ ocasional, $\mathrm{A}=\mathrm{a}$ menudo, $\mathrm{M}=$ moderada, $\mathrm{I}=$ intensa, $\mathrm{P}=$ puntaje, $\mathrm{S}=$ severidad.

Este cuestionario valora la frecuencia e intensidad de los síntomas oculares y extraoculares característicos del Síndrome Visual Informático; consta de 16 ítems, a cada uno de ellos se le asigna un puntaje de acuerdo con su frecuencia de aparición y otro por su intensidad. La multiplicación de estos puntajes se codificará para determinar severidad de cada uno de los síntomas. Si la sumatoria de los puntajes de severidad es mayor a 6 se considera que el trabajador presenta Síndrome Visual Informático (Seguí, 2015).

\section{Evaluación del riesgo ergonómico con el método Rapid Office Strain Assessmentt (ROSA)}

Para implementar el programa de prevención del Síndrome Visual Informático, se realizó una evaluación anterior y posterior a las mejoras, mediante el método Rapid Office Strain Assessment (ROSA). Se escogió este método por cuanto evalúa los riesgos ergonómicos que predominan durante el teletrabajo y están relacionados con la aparición del SVI. El 
método ROSA, consta de una lista de verificación aplicable a los puestos en los que el trabajador permanece sentado, haciendo uso de pantallas de visualización de datos (Diego-Mas, 2015). Evalúa los elementos utilizados más comúnmente: la silla, la pantalla y los periféricos (teclado, mouse y teléfono); el tiempo de uso de cada uno de éstos puede aumentar o disminuir la puntuación obtenida. La puntuación total varía entre 1 y 10 , incrementando de manera directa con el nivel de riesgo, y siendo el puntaje mayor a 5 el indicador de riesgo elevado (Diego-Mas, 2015) Ver tabla 2. Además, este método permite obtener una estimación de la necesidad de actuar o no, dependiendo del nivel de riesgo resultante. Ver tabla 5. (Diego-Mas, 2015).

Tabla 2

Rapid Office Strain Assessment (ROSA)

\begin{tabular}{|c|c|c|}
\hline No & ITEM & PUNTAJE \\
\hline \multirow[t]{7}{*}{1} & ALTURA DEL ASIENTO & \\
\hline & Rodillas flexionadas $90^{\circ}$ (aproximadamente) & 1 \\
\hline & Asiento muy bajo, rodillas $<90^{\circ}$ & 2 \\
\hline & Asiento muy alto, rodillas $>90^{\circ}$ & 2 \\
\hline & Pies sin contacto con el suelo & 3 \\
\hline & Puntúa extra: Espacio insuficiente para las piernas bajo la mesa & +1 \\
\hline & Altura del asiento no regulable & +1 \\
\hline \multirow[t]{5}{*}{2} & PROFUNDIDAD DEL ASIENTO & \\
\hline & $8 \mathrm{~cm}$ (aprox) de espacio entre el asiento y la parte trasera de las rodillas & 1 \\
\hline & Asiento muy largo $(<8 \mathrm{~cm})$ & 2 \\
\hline & Asiento muy corto $(>8 \mathrm{~cm})$ & 2 \\
\hline & Puntúa extra: La profundidad del asiento no es regulable & +1 \\
\hline \multirow[t]{7}{*}{3} & REPOSABRAZOS & \\
\hline & Codos bien apoyados, hombros relajados & 1 \\
\hline & Reposabrazos muy altos, hombros encogidos & 2 \\
\hline & Reposabrazos muy bajos, los codos no se apoyan & 2 \\
\hline & Puntúa extra: Reposabrazos demasiado separados & +1 \\
\hline & Superficie del reposabrazos dura o dañada & +1 \\
\hline & Reposabrazos no ajustables & +1 \\
\hline \multirow[t]{6}{*}{4} & RESPALDO & \\
\hline & Respaldo reclinado $95-110^{\circ}$, apoyo lumbar adecuado & 1 \\
\hline & Sin apoyo lumbar o situado en la parte baja de la espalda & 2 \\
\hline & Respaldo reclinado menos de $95^{\circ}$ o más de $110^{\circ}$ & 2 \\
\hline & Puntúa extra: Sin respaldo & +1 \\
\hline & Respaldo no ajustable & +1 \\
\hline
\end{tabular}


Tabla 2

Rapid Office Strain Assessment (ROSA) (continuación)

\begin{tabular}{llc}
\hline No & \multicolumn{1}{c}{ ITEM } & PUNTAJE \\
\hline 5 & PANTALLA & 1 \\
\hline & Pantalla a $45-75 \mathrm{~cm}$ de distancia de los ojos, borde superior a la altura de los ojos. & 2 \\
& Pantalla muy baja, $30^{\circ}$ por debajo de los ojos & 3 \\
& Pantalla muy alta, provoca extensión del cuello & +1 \\
& Puntúa extra: Pantalla muy lejos & +1 \\
Pantalla desviada lateralmente, es necesario girar el cuello & +1 \\
Brillos o reflejos en la pantalla & -1 \\
Tiempo de uso diario menor a 1 hora & 0 \\
Tiempo de uso diario de 1-4 horas & +1 \\
Más de 4 horas de uso diario &
\end{tabular}

6 TELEFONO

Uso de auriculares, o se usa el teléfono con una mano y el cuello en posición

neutral.

El teléfono está lejos

Puntúa extra: El teléfono se sujeta entre el cuello y el hombro $\quad+2$

El teléfono no tiene función manos libres $\quad+1$

Tiempo de uso diario menor a 1 hora $\quad-1$

Tiempo de uso diario de 1-4 horas $\quad 0$

Más de 4 horas de uso diario $\quad+1$

7 MOUSE

El mouse está alineado con el hombro

1

El mouse no está a lineado con el hombro 2

Puntúa extra: El mouse y el teclado están a diferente altura $\quad$ +2

Mouse muy pequeño $\quad+1$

Existen puntos de presión en la mano al usar el mouse $\quad+1$

Tiempo de uso diario menor a 1 hora $\quad-1$

Tiempo de uso diario de 1-4 horas 0

Más de 4 horas de uso diario $\quad+1$

8 TECLADO

Las muñecas están rectas y los hombros relajados 1

Las muñecas están extendidas más de $15^{\circ} \quad 2$

Puntúa extra: Las muñecas están desviadas lateralmente hacia dentro o fuera $\quad+1$

El teclado está demasiado alto, los hombros encogidos $\quad+1$

El teclado, o la plataforma sobre la que reposa, no son ajustables $\quad+1$

Tiempo de uso diario menor a 1 hora $\quad-1$

$\begin{array}{ll}\text { Tiempo de uso diario de 1-4 horas } & 0\end{array}$ 
Nota: Ítems del Cuestionario Rapid Office Strain Assessment (ROSA). Adaptado de (DiegoMas, 2015)

\section{Tabla 3}

Nivel de Actuación según la puntuación final obtenida

\begin{tabular}{|c|c|c|c|}
\hline PUNTUACIÓN & RIESGO & NIVEL & ACTUACIÓN \\
\hline 1 & Inapreciable & 0 & No es necesaria actuación. \\
\hline $2-3-4$ & Mejorable & 1 & $\begin{array}{l}\text { Pueden mejorarse algunos elementos del } \\
\text { puesto. }\end{array}$ \\
\hline 5 & Alto & 2 & Es necesaria la actuación. \\
\hline $6-7-8$ & Muy Alto & 3 & Es necesaria la actuación cuanto antes. \\
\hline $9-10$ & Extremo & 4 & Es necesaria la actuación urgentemente. \\
\hline
\end{tabular}

Nota: Ítems del Cuestionario Rapid Office Strain Assessment (ROSA). Adaptado de (DiegoMas, 2015)

\section{Resultados}

\section{Resultado de la aplicación del cuestionario Computer Vision Syndrome Questionnaire (CVS-Q)}

Luego de aplicar el CVS-Q, se observó que el 76\% de los docentes encuestados presentan Síndrome Visual Informático, siendo más predominante su presencia en el personal femenino, y dentro del rango de edad de 20 a 35 años. Ver figura1.

\section{Figura 1}

Resultados CVS-Q - presencia SVI

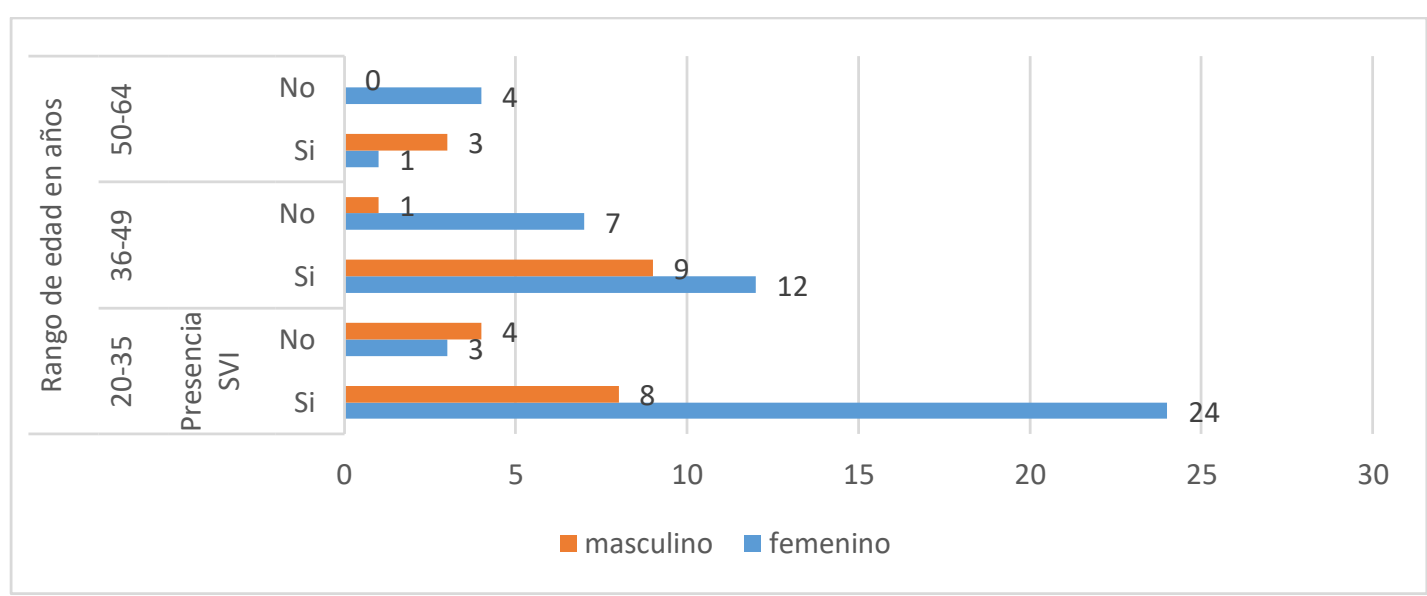

Elaborado por: autor

\section{Resultado riesgo ergonómico con el método Rapid Office Strain Assessment (ROSA)}

Al aplicar el método ROSA, se evidenció que el $61.3 \%$ de los docentes se encontraban expuestos a niveles de riesgo alto y muy alto. No se encontró trabajadores expuestos a riesgo extremo, ni trabajadores con puestos de trabajo óptimos. Ver figura 2. 


\section{Figura 2}

Resultados de la aplicación del Método ROSA - Nivel de riesgo ergonómico

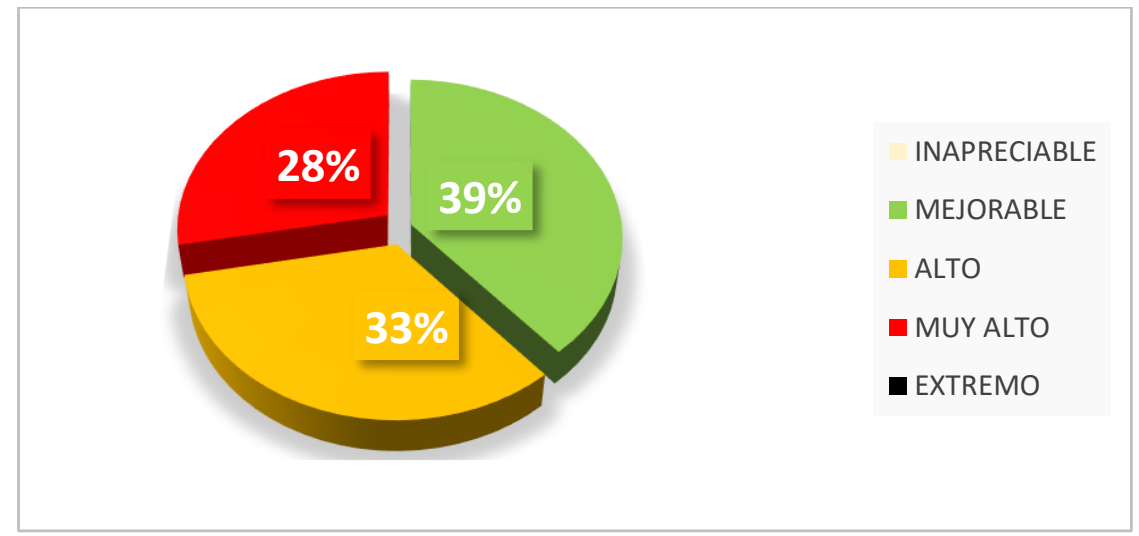

Elaborado por: autor

Al relacionar el nivel de riesgo ergonómico con la presencia del Síndrome Visual informático, se pudo determinar que el $92 \%$ de trabajadores expuestos a riesgo ergonómico alto durante el teletrabajo presentaban esta patología, mientras que, en aquellos expuestos a riesgo muy alto, el porcentaje de la misma correspondía al $90.5 \%$. Ver figura 3.

\section{Figura 3}

Asociación entre SVI y Riesgo ergonómico - método ROSA

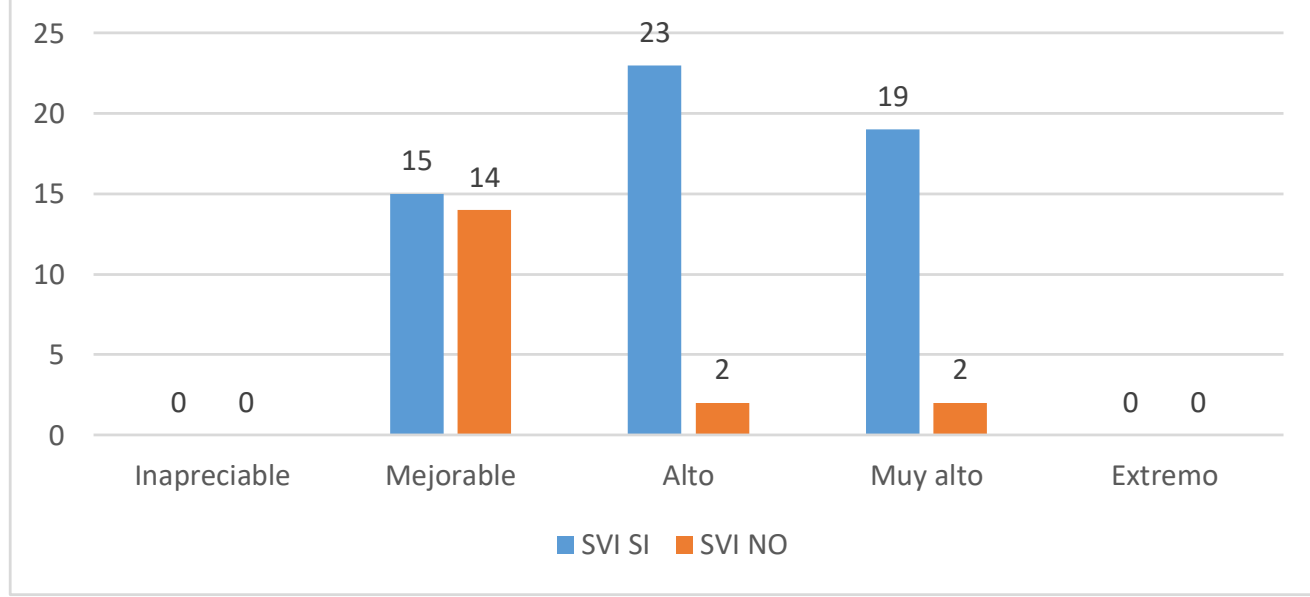

Elaborado por: autor

Con estos resultados se realizó una capacitación a los trabajadores, durante la cual se determinó la factibilidad de modificar ciertas condiciones del puesto de trabajo, tales como: tiempo de exposición a pantalla y periféricos, altura del asiento, inclinación del respaldo y apoyo lumbar, posición de la pantalla, brillos y reflejos en la pantalla, uso del teléfono y uso del mouse. Con estas adecuaciones se evidenció una importante disminución del nivel de riesgo ergonómico. Ver figura 4. 


\section{Figura 4}

Variación del nivel de riesgo tras aplicación de medidas correctivas

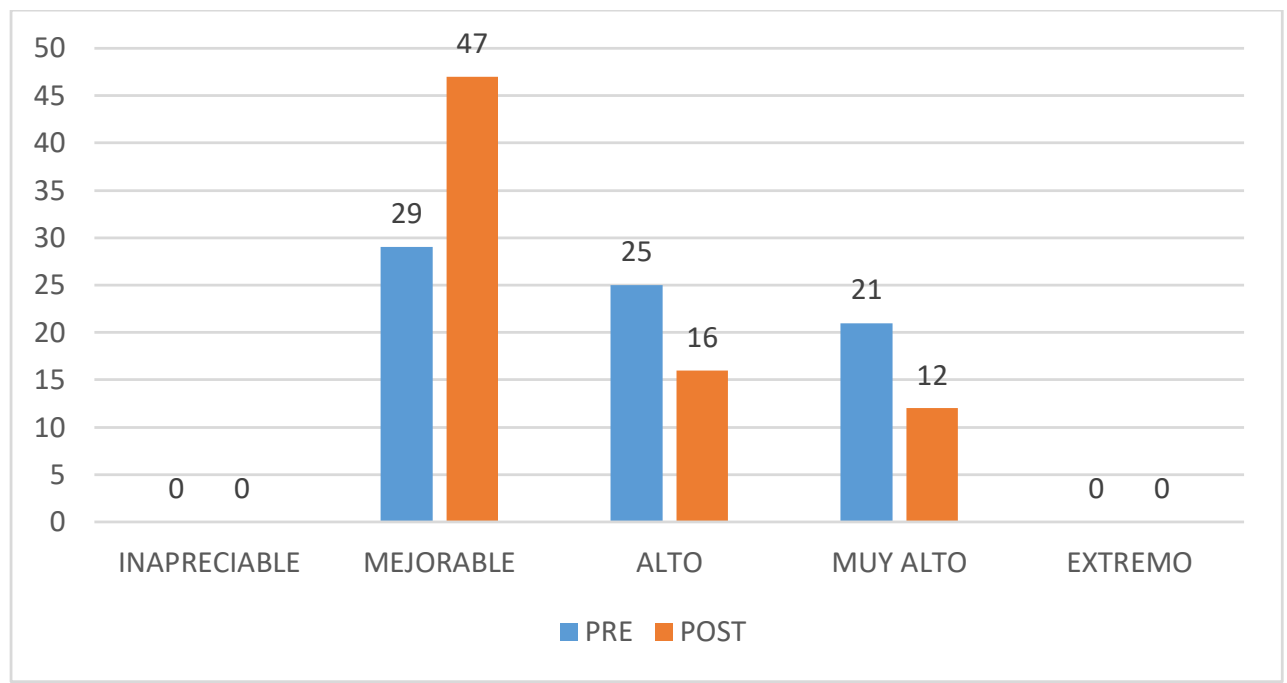

Elaborado por: autor

\section{Discusión}

Se observó que las mujeres y quienes se encuentran dentro del grupo etario entre 20 y 35 años, son quienes presentan mayor prevalencia de Síndrome Visual Informático. Es posible que esto esté relacionado a una mayor exposición a PVD por su uso continuo por motivos recreativos o extra laborales.

Se puede concluir que los factores ocupacionales como el tiempo frente a la pantalla, distancia entre el usuario y la PVD, y el uso de periféricos (mouse, teclado, teléfono), tienen una asociación significativa con la presencia de esta patología.

El enfoque más importante en el manejo es eliminar el factor causal que conduce a los síntomas. Las medidas preventivas deben incluir la modificación de factores ambientales y el autocuidado adecuado de la vista por parte del trabajador (Loh \& Redd, 2008). La distancia adecuada de la pantalla, el ajuste adecuado del tamaño de la imagen y la altura adecuada del asiento son factores importantes a considerar, así como la adecuación de los periféricos. Se recomienda que los ojos estén a unas 35-40 pulgadas de la pantalla y que la pantalla se coloque 10-20 grados por debajo o que el centro de la pantalla 5-6 pulgadas por debajo del nivel de los ojos (Loh \& Redd, 2008).

La sequedad ocular secundaria a la disminución de la frecuencia de parpadeo se puede controlar fácilmente aplicando gotas oculares lubricantes o lágrimas artificiales. Se alienta a los trabajadores que tienen síntomas recurrentes del síndrome de visión por computadora a que se sometan a una revisión y evaluación adecuadas por parte de un optometrista. 
En quienes no se ha desarrollado el síndrome, se deben fomentar las medidas preventivas para evitarlo (López et al., 2020).

\section{Conclusiones}

- El 92\% de trabajadores están expuestos a riesgo ergonómico alto durante el teletrabajo y presentan síndrome visual informático, mientras que, en aquellos que están expuestos a riesgo muy alto les corresponde un $90.5 \%$ en la Unidad Educativa.

- La adecuación del puesto de trabajo con la implementación de pausas activas, gimnasia ocular, tiempo de exposición a pantalla, uso correcto de periféricos, Uso de silla ergonómica, posición de la pantalla, uso de pantalla antirreflejos, uso del mouse ergonómico, disminuye el riesgo ergonómico en un $100 \%$ según el método ROSA.

\section{Referencias bibliográficas}

America Optometric Association. (30 de 3 de 2021). American Optometric Association. Obtenido de Computer Vision Syndromre: https://www.aoa.org/patients-andpublic/caring-for-your-vision/protecting-your-vision/computer-vision-syndrome

Diego-Mas, J. A. (2015). Ergonautas. (U. P. Valencia, Ed.) Recuperado el 21 de 09 de 2021, de http://www.ergonautas.upv.es/metodos/rosa/rosa-ayuda.php

Instituto de Seguridad y Salud en el Trabajo. (2020). NTP 1150. Riesgos ergonómicos en el uso de las nuevas tecnologías con pantallas de visualización.

Loh, K., \& Redd, S. (2008). Understanding and preventing computer vision syndrome. Malaysian Family Physician, 128-130.

López-Camones, J., Rojas-Meza, L., \& Osada, J. (2020). Frecuencia de factores ocupacionales asociados a astenopía en trabajadores usuarios de pantallas de visualización de datos de empresas del rubro construcción en Huaraz, 2019. Revista Asociación Española de Medicina del Trabajo, 29(2), 56-66.

Ministerio de Educación del Ecuador. (s.f.). ACUERDO Nro. MINEDUC-MINEDUC2020-00020-A20. Obtenido de https://educacion.gob.ec/wpcontent/uploads/downloads/2020/04/MINEDUC-MINEDUC-2020-00020-A.pdf

Organización Internacional del Trabajo [OIT]. (2020). El teletrabajo durante la pandemia de COVID-19. Ginebra. Obtenido de www.ilo.org/publns

Organización Internacional del Trabajo [OIT]. (2020). Guía para empleadores sobre el trabajo desde casa en respusta al brote de la COVID-19.

Organización Internacional del Trabajo [OIT]. (2020). El teletrabajo durante la pandemia de COVID-19 y después de ella. Ginebra. 
Prado Montes, A., Morales Caballero, Á., \& Jossias, M. C. (2017). Síndrome de fatiga ocular y su relación con el medio laboral. Medicina y Seguridad del Trabajo, 345361.

Seguí, M. d. (2015). A reliable and valid questionnaire was developed to measure computer visión syndrome at the workplace. Journal of Clinical Epidemiology, 68(6), 662-673. doi:https://doi.org/10.1016/j.jclinepi.2015.01.015

Serra, F. (2020). El teletrabajo - implicancias en la seguridad y salud en el trabajo. CADE, 35-43. Obtenido de https://prevyso.com.uy/wpcontent/uploads/2020/09/FRANCISCO-SERRA-EL-TELETRABAJO-08.20.pdf

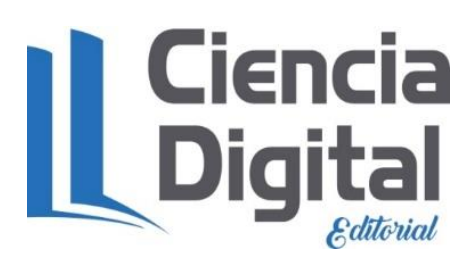




\section{PARA CITAR EL ARTÍCULO INDEXADO.}

Nieto Paredes, F., \& Córdova Suárez, M. A. (2021). Prevención del síndrome visual informático en docentes que realizan teletrabajo en una unidad educativa . Anatomía Digital, 4(3.1), 163-174. https://doi.org/10.33262/anatomiadigital.v4i3.1.1918

\section{Liencia}

El artículo que se publica es de exclusiva responsabilidad de los autores y no necesariamente reflejan el pensamiento de la Revista Anatomía Digital.

El artículo queda en propiedad de la revista y, por tanto, su publicación parcial y/o total en otro medio tiene que ser autorizado por el director de la Revista Anatomía Digital.
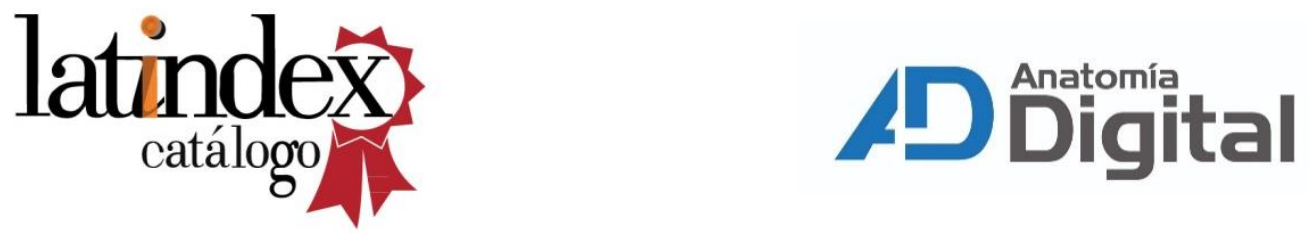